\title{
Literaturwissenschaft
}

\author{
Aleksandra Wojnarowska \\ Ernst-Moritz-Arndt Universität, Greifswald
}

DOI: $10.19195 / 0435-5865.141 .1$

\section{Über den Einfluss der Aerostatik auf die Literatur um 1800 oder das naturwissenschaftliche Potential der Münchhausiade}

„Wer kennt nicht die scherzhaften Erzählungen des Herrn von Münchhausen, die mit einer so monströsen Phantasie zusammengesetzt sind, daß man sie komische Arabesken nennen könnte?"1 - so die rhetorische Frage auf den Seiten der ersten Auflage des Brockhaus Conversations-Lexikons im Jahre 1809 unter dem Stichwort Des Freiherrn von Münchhausen. ${ }^{2}$ Die Abenteuer des Lügenbarons wurden im Laufe der Zeit in mehrere Sprachen übersetzt sowie mehrfach verfilmt und sind auch heute noch weltweit bekannt. ${ }^{3}$ Die Münchhausiaden liefern nicht nur unterhaltende, weil unglaubwürdige, Jagd- und Kriegsgeschichten, sondern ermöglichen ihren Leserinnen und Lesern bis heute einen Einblick in die Vorstellungswelt um das Jahr 1800 herum. Dies gilt in besonderer Weise für die sich damals allmählich entwickelnde Naturforschung. ${ }^{4}$ In den Abenteuern Münchhausens lassen sich zahlreiche Anspielungen sowohl auf technische Neuentwicklungen um 1800

${ }^{1}$ Brockhaus Conversations-Lexikon Bd. 3. Amsterdam 1809, S. 194-195.

${ }^{2}$ Fast hundert Jahre später findet man im Brockhaus die folgenden Informationen zu Münchhausen: „Münchhausen, Karl Friedr. Hieronymus, Freiherr von, geb. 11. Mai 1720 auf Bodenwerder (Hannover), eine Zeit Offizier in russ. Diensten, gest. 22. Febr. 1797; bekannt durch seine lügenhaften Erzählungen von Jagd- und Kriegsabenteuern; engl. Bearbeitung (1785) von Raspe (geb. 1737, gest. 1794 in London), deutsch von Bürger (1787; neue Ausg. 1890). - Vgl. Ellißen (11. Aufl. 1890). - Münchhausiāden, grotesk-komische Aufschneidereien." Brockhaus’ Kleines Konversations-Lexikon, fünfte Auflage, Band 2. Leipzig 1911, S. 225.

${ }^{3}$ Vgl. Wiebel, Bernhard: Münchhausen - das Märchen vom Lügenbaron, in: Bendix, Regina / Marzolph, Ulrich: Hören, Lesen, Sehen, Spüren: Märchenrezeption im europäischen Vergleich. Hohengehern: Schneider Verlag 2008, S. 47-49.

${ }^{4}$ Beese, Melanie: Münchhausens wunderbare wissenschaftliche Abenteuer zu Wasser und in der Luft und wie er diese zu erzählen pflegt. Eine literatur- und wissensgeschichtliche Studie. Bielefeld: Aisthesis Verlag 2014. 
(Fahrzeuge wie Wasserstoffballons, Kriegsschiffe oder Dampfschiffe) als auch auf politische und gesellschaftliche Prozesse sowie Umwälzungen der Zeit (wie zum Beispiel die Kolonialpolitik oder die Durchführung von damals noch neuartigen demokratischen Wahlen) finden. ${ }^{5}$

Die Anekdoten über den Baron Hieronymus Carl Friedrich Freiherr von Münchhausen (1720-1797) wurden zuerst mündlich verbreitet und erst im Jahre 1781 in Berlin als Vademecum für lustige Leute anonym herausgegeben. Der in England lebende deutsche Schriftsteller Rudolf Erich Raspe (1736-1794) veröffentlichte kurz danach auch anonym seine überarbeitete Form der Erzählungen aus dem Vademecum in der englischen Sprache als Baron Munchhausens Narrative of His Marvellous Travels und Campaigns in Russia. Im Laufe der Zeit gibt Raspe mehrere Auflagen heraus, überarbeitet sie und erweitert die Anekdoten um die Seeabenteuer des Barons. Im Jahre 1786 veröffentlicht Gottfried August Bürger die deutsche Version der Erzählungen. Wunderbare Reisen zu Wasser und zu Lande - Feldzüge und lustige Abenteuer des Freiherrn von Münchhausen von Bürger sind im Grunde eine frei übersetzte und erweiterte Version von Raspes Münchhausen.

In der literaturwissenschaftlichen Forschung herrscht im Allgemeinen die Überzeugung vor ${ }^{6}$, dass insbesondere die naturwissenschaftlichen Aspekte der Anekdoten Münchhausens (sowohl in der englischen Version von Raspe als auch in der deutschen von Bürger) nach wie vor ein unentdecktes Feld darstellten. In diesem Bereich stehen meines Wissens lediglich die Studie von Bachmann-Medick $^{7}$ sowie jüngst eine Veröffentlichung von Beese zur Verfügung. In dieser weist Beese darauf hin, dass Gottfried August Bürgers Münchhausenversion die Abenteuererzählungen nicht nur um inhaltliche Aspekte erweiterte, sondern ihnen auch eine neue literarische Form gab. ${ }^{8}$ So konstatiert die Autorin, dass für die englische Münchhausenversion von Raspe eine gewisse sprachliche Nüchternheit charakteristisch sei, die an die Sachlichkeit von naturwissenschaftlichen Darstellungen erinnere. Die Version Bürgers sei hingegen ,volkstümlicher poetisch“. 9 Sie basiere auf ,publikumswirksame[n] Fiktionalisierungen "10 und - was damit einhergeht sei häufiger wertend und moralisierend. ${ }^{11}$ Dementsprechend charakterisiert Bachmann-Medick die Version Bürgers als eine „einbürgernde Übersetzung“ ${ }^{12}$ welche häufiger Anspielungen auf das damalige Volkswissen beinhalte und welches an

${ }^{5}$ Siehe dazu: Beese (2014).

${ }^{6}$ Ebd. (2014: 14).

${ }^{7}$ Bachmann-Medick, Doris: Fremddarstellung und Lüge: Übersetzung als kulturelle Übertreibung am Beispiel von Münchhausens Lügengeschichten, in Bachmann-Medick, Doris (Hrsg.): Übersetzung als Repräsentation fremder Kulturen. Berlin: Erich Schmidt Verlag 1997, S. 42-68.

${ }^{8}$ Beese (2014: 170)

9 Bachmann-Medick (1997: 47).

${ }^{10}$ Ebd., S. 50.

11 Bachmann-Medick (1997: 50).

12 Ebd., S. 47. 
den „deutsche[n] Erfahrungshorizont“13 anknüpfe. Diesem Befund entspricht, dass sich sowohl Bachmann-Medick als auch Beese in ihren Analysen vor allem auf die englischsprachigen Verarbeitungen des Münchhausenstoffes konzentrieren, weil sich in diesen die Spuren der Naturforschung deutlicher herauslesen ließen - beispielsweise als Gebrauch von Fußnoten und Fachwörtern, wie sie in wissenschaftlichen Veröffentlichungen üblich sind. ${ }^{14}$ In Bürgers Fassung hingegen würden wissenschaftliche Inhalte eher verschleiert wiedergegeben. ${ }^{15}$ Jedoch lässt sich in seiner Version - so meine Ausgangsthese - dennoch beobachten, wie Elemente der Naturforschung auf die erzählerische Ebene übertragen wurden.

In den folgenden Textabschnitten wird zunächst die Handlung von Münchhausens Viertem Seeabenteuer, in deren Zentrum eine Fahrt mit einem Wasserstoffballon steht, umrissen. Daran anschließend wird eine literaturwissenschaftliche Analyse des Textes durchgeführt, die sich nicht lediglich auf die Hauptfigur der fiktional-literarischen Erzählung fokussiert. Stattdessen soll gezeigt werden, wie die Münchhausiade den Wissenstransfer zwischen Naturforschung und Literatur um 1800 erkennen lässt. Als weiterer Schritt - um nicht in die Falle einer analytischen Einbahnstraße ${ }^{16}$ zu geraten - wäre es eigentlich sinnvoll zu überlegen, inwiefern in umgekehrter Richtung die Naturforschung durch die literarische Produktion um 1800 beeinflusst wurde, um sicherzugehen, dass die aktive Rolle der Literatur in diesem wechselseitigen Prozess nicht außer Acht gelassen wird. Dies würde jedoch den Rahmen des vorliegenden Aufsatzes sprengen. Ich konzentriere mich deshalb in dieser Untersuchung bewusst zunächst ${ }^{17}$ auf eine Betrachtung des gerichteten Einflusses, den die Naturforschung auf die Literatur um 1800 zeitigte. Die Analyse nimmt hierbei die Ebene von (diegetischem) Raum und Zeit in den Fokus, weil sich - wie gezeigt werden soll - am Beispiel dieser Elemente besonders gut beobachten lässt, wie naturwissenschaftliche Strukturen, zum Beispiel die Praxis des naturwissenschaftlichen Experiments, in der erzählten Welt der Münchhausenfassung von Bürger weiter präsent bleiben.

\section{Zur Handlung}

Im Kapitel Viertes Seeabenteuer von Bürgers Münchhausen begegnet der Rezipient dem Lügenbaron, als dieser sich ,im türkischen Dienste“ befindet. Münchhausen bewundert gerade aus einer Lustbarke heraus den Himmel über Istanbul,

13 Ebd.

${ }^{14}$ Vgl. Beese (2014: 170).

15 Ebd.

16 Siehe dazu: Pethes 2004: 353. Ein Überblick über die Forschungsliteratur zum Transfer zwischen Naturwissenschaft und Literatur findet sich bei: Pethes 2003.

${ }^{17}$ In meiner voraussichtlich 2018 erscheinenden Dissertationsschrift wird eine Analyse beider Richtungen dargelegt werden. 
als er plötzlich „ein rundes Ding, ohngefähr wie eine Billardkugel groß, in der Luft" schweben sieht. Die Figur greift - scheinbar ohne sich viele Gedanken zu machen - zu seiner Flinte, lädt sie (nomen est omen mit „Kugeln“"18) und versucht, die fliegende Kugel vom Himmel zu schießen. Erst nach dem dritten Schuss gelingt es ihm „das Ding“, welches sich schließlich als ein Wasserstoffballon mit einem Franzosen an Bord entpuppt, herabzubringen.

Der abgestürzte Fahrer erzählt dem Baron die Geschichte seines Fluges: er war vor ca. sieben oder acht Tagen in England in die Luft gestiegen, um einer Zuschauermenge mit dem Ballon ein Schauspiel zu geben. Jedoch verlor er schon bald nach dem Aufstieg die Kontrolle über sein Fluggerät. Die Winde trieben den Ballonfahrer so lange fort, bis er schließlich von Münchhausens Flinte gerettet (a.w) wurde. Während seiner vom Wind gelenkten Fahrt war er gezwungen, sich vom Fleisch eines Schafes zu ernähren, welches ursprünglich Bestandteil des „Kunststückes“ war. ${ }^{19}$ Dazu kam es jedoch nie, weil noch vor der Aufführung des Spektakels die Kontrolle über den Ballon verlustig ging. ${ }^{20}$ Das Schaf ging daher mit auf die Reise und rettete dem menschlichen Balloninsassen letztlich das Leben, da er es schlachten und in der Sonne braten konnte. Erst gegen Ende seines Berichts kommt der Franzose auf die eigentliche Ursache seiner langen Reise zu sprechen: der Ballonfaden zerriss und die Maschine leichter als Luft war fortan nicht mehr lenkbar.

\section{Analyse des Textes}

Mit der topologischen Opposition zwischen oben (der Ballon am Himmel) und unten (die Figur Münchhausens auf der Erde) ist auf den ersten Blick ein semantischer Gegensatz verbunden, nämlich der Dualismus Nicht-Wissen vs. Wissen. ${ }^{21}$ So weiß

${ }^{18}$ In einem verhältnismäßig kurzen Text, der aus 731 Wörtern besteht, finden sich 23 Bezeichnungen, die runde Gegenstände umschreiben, unter anderen: „Kugel“, „Sonne“, „Mond“, „Ballon“, „Medaille“, „Knopfloche“, „Ring“, „Auge“ usw. Auf eine analoge Anhäufung von ,runden Gegenstandsbezeichnungen' in einem anderen literarischen Text zur Ballonfahrt, nämlich: Jean Pauls Des Luftschiffers Giannozzo Seebuch, macht Juliane Vogel aufmerksam: Luftkugel und fliegendes Auge, in: Stadler, Ulrich / Wagner, Karl (Hrsg.): Schaulust. Heimliche und verpönte Blicke in Literatur und Kunst. Paderborn: Fink 2005, 55-72.

${ }^{19}$ Es lässt sich feststellen, dass in Münchhausens Abenteuern Tiere häufig nur als Mahlzeit (siehe z.B. die Enten an der Leine oder die blinde Bache in Bürgers Kapitel „Jagdgeschichten“) oder auch als reine Trophäen betrachtet wurden, welche man zum Spaß tötet. So beispielsweise der Wolf, der von der Figur Münchhausen umgekrempelt wurde oder der Bär (ebenfalls im zweiten Kapitel: „Jagdgeschichten“). Die Darstellung des Schafes im vierten Seeabenteuer unterscheidet sich also nicht stark von den anderen Tierdarstellungen in den Erzählungen über Münchhausen. Das Schaf dient erst dem Spiel und Spaß (,Kunststücke“) und wird danach verspeist.

${ }^{20}$ In Raspes Fassung möchte der Franzose Experimente in der Luft durchführen, er ist aber „too much high to make observations“, Siehe: Beese (2014: 42-43).

${ }^{21}$ Relevant ist hier der Unterschied zwischen dem Nicht-Wissen des potentiellen Rezipienten, der den Raum der Fiktion also die Möglichkeiten zur weiteren Entfaltung der erzählten Welt eröffnet und dem Nicht-Wissen der Figur Münchhausens über das, was in der Luft vor ihm schwebt. 
der am Boden befindliche Lügenbaron zu Anfang nicht genau, worum es sich bei dem ,runden Ding“ eigentlich handelt. ${ }^{22}$ Der Franzose scheint demgegenüber das Wissen zu symbolisieren, da vorausgesetzt werden kann, dass er über einige Kenntnis verfügen muss, um das moderne Fluggerät in die Lüfte steigen lassen und manövrieren zu können. Der Ballonfahrer erscheint dem Ich-Erzähler jedoch nur auf den ersten Blick als kompetente und hochrangige Persönlichkeit. Er wird satirisch beschrieben: „Aus jedem Knopfloche hing ihm eine goldene Medaille, wenigstens hundert Dukaten am Wert, und an jeglichem seiner Finger steckte ein kostbarer Ring mit Brillanten“. ${ }^{23}$ Jedoch entpuppt sich das Wissen des Ballonfahrers schon bald als bloßer Schein - er gibt zu, das Gerät nicht selbst erfunden und gebaut („Dieses Luftfuhrwerk hatte ich zwar nicht Kopf und Wissenschaft genug selbst zu erfinden, dennoch..."24) und außerdem die Kontrolle darüber verloren zu haben. Sein Wissen erscheint Münchhausen daher nicht mehr wert zu sein als die Kenntnis von „Luftspringer- und Seiltänzerwaghalsigkeit[en]“.${ }^{25}$ Der Luftfahrt wird im Vierten Seeabenteuer kein aufklärerisches Moment, ${ }^{26}$ kein technischer Fortschritt zugestanden - vielmehr wird sie als zirkusähnliches Kunststück beschrieben.

Wissen und Nicht-Wissen der Figur Münchhausen ist semantisch mit einem weiteren Gegensatzpaar verbunden, nämlich mit der topographischen Opposition Orient vs. Okzident: zu Beginn der Erzählung sieht die Figur das heranschwebende Objekt als kleines, weit entferntes und unbekanntes Ding, welches in die Welt des Orients eindringt. Das fliegende Objekt ist also zu Anfang sehr weit entfernt und ist in gewisser Weise verkleinert. Die Verkleinerung des Objekts erinnert an den wissenschaftlichen Blick durch das Fernrohr oder das Mikroskop. Der runde Gegenstand taucht hierbei erstmalig vor dem Hintergrund des blauen Himmels sowie der türkischen Bauten auf (Inbegriffe des Morgenlandes: der Serail des Großsultans, prachtvolle Gebäude, Minaretten). Die Figur Münchhausens sieht sich diesem Panoramabild (der Komposition aus Himmel und orientalischer Welt) zu Anfang frontal gegenübergestellt und bewundert den Anblick aus seiner Lustbarke heraus. Plötzlich wird der idyllische Rundblick durch ein fremdes rundes Objekt gestört. Statt jedoch zu einem Fernrohr zu greifen, um sich das nähernde Unbekannte zunächst zu besehen, greift Münchhausen sogleich zu seiner „besten und längsten Vogelflinte" und attackiert das Objekt brutal. Münchhausen assoziiert das plötzlich aufgetauchte Unbekannte inmitten der morgenländischen Welt als etwas, das geheimnisvoll und potentiell gefährlich sein kann. Der Kontrast zwischen dem plötzlichen Auftauchen des Wasserstoffballons und der zuvor herrschenden Idylle zeugt davon, wie widersprüchlich der Orient um 1800 wahrgenommen und in der

${ }^{22}$ Seinem Verhalten nach, nämlich dem Aufheben seiner Vogelflinte, lässt sich freilich auch schlussfolgern, dass er eigentlich denkt, dass es sich bei dem Ballon um einen Vogel handelt.

23 Bürger (1981 [1786]: 45).

${ }^{24} \mathrm{Ebd}$.

25 Ebd.

${ }^{26}$ Ebd. 
Literatur dargestellt wurde: einerseits wird er als geradezu langweilig ${ }^{27}$ ruhig und harmonisch und - in diesem Sinne - als vorhersehbar beschrieben. Andererseits verwandelt sich diese Welt mit dem Auftauchen des Ballons schlagartig in einen Ort, an dem sich eine Vielfalt von Geheimnissen und Gefahren zu verbergen scheint. Das Morgenland gilt im Text nicht als Raum des Wissens, sondern als ein Raum des Nicht-Wissens - in diesem Raum ist nichts naturwissenschaftlich erwiesen, auf die Menschen warten Überraschungen, die potentiell gefährlich sind. Im Orient scheint kein naturwissenschaftliches Wissen zu herrschen, sondern eher ein irrationales Volkswissen, welches einen Raum konstruiert, in dem sich der Mensch vor unbekannten fliegenden Objekten hüten muss.

Hierin zeigt sich deutlich ein Bild des Orients, wie es von der deutschen Literatur des 18. Jahrhunderts gezeichnet wurde. Beispielsweise macht Polaschegg darauf aufmerksam, dass das Morgenland um 1800 als „Land [der] nahezu unbegrenzten Möglichkeiten“ 28 charakterisiert wurde. Der Wasserstoffballon verstärkt hierbei die Ambivalenz des Orients sowie die Kluft zwischen dem Morgen- und dem Abendland mit seiner eigenen inneren Widersprüchlichkeit: er selbst vereint in sich mehrere polarisierte diskursive Positionen ${ }^{29}$ - so insbesondere die Gegensätze Natur vs. Kultur, unseriöses Jahrmarktspektakel vs. experimentelle ${ }^{30}$ (mit Fortschritt assoziierte „Frucht des Genies“31) Versuchsanordnung sowie weibliche Schaulust vs. männliche kalte Vernunft - und verstärkt durch sein Auftauchen die Opposition zwischen geheimnisvollem Orient und fortschrittlichem Abendland.

Im Laufe der Handlung verändert sich die Perspektive, in welcher der Rezipient die erzählte Welt wahrnimmt: das Objekt wird immer größer (Vergrößerung in einem Mikroskop oder unter einer Lupe) und entpuppt sich letztlich als, nicht sehr fremdländischer, okzidentaler Wasserstoffballon. Der Ballon wird hierbei einerseits als potentielle Gefahr in die Handlung eingeführt, die zu Anfang aus der Entfernung langsam heranschwebt und noch undeutlich und fremd bleibt. Andererseits wird der Ballon nach seiner Landung als Repräsentant der okzidentalen Welt identifiziert und somit als ein dem Ich-Erzähler vertrautes Objekt. Es ist zu betonen, dass die räumliche Perspektive ${ }^{32}$ bei diesem Vorgang dynamisch bleibt. Der weite Blickwinkel der Panorama-Aufnahmen des Erzählanfangs ändert sich nicht erst allmählich. Vielmehr verändert sich der Blickwinkel des Ich-Erzählers äußerst abrupt, wenn vermittels der Übergänge vom anfänglichen Rundblick zur Wahrnehmung des kleinen Objekts in der Ferne hin zum Auftauchen des dann

${ }^{27}$ So langweilig, dass Münchhausen eine Lustbarke besteigt, um nach „Belustigung“ zu suchen.

28 Polaschegg (2008: 35).

${ }^{29}$ Siehe dazu: Link (1984: 151-152) sowie Link (1988: 290-296).

30 Ebd.

${ }^{31}$ Faujas de Saint-Fond (1784: Einleitung, S. XII).

32 Die räumliche Perspektive ist hierbei nicht mit der Perspektivierung im Sinne von „Modus“ zu verwechseln (Martinez/Scheffel 2012: 49-66). Gemeint ist an dieser Stelle der diegetische Raum in der erzählten Welt. 
groß erscheinenden Ballons (,größer als die größte Turmkuppel im Umfange“ $)^{33}$ schließlich eine Fokussierung auf das Objekt erreicht wird.

Mithilfe der Einführung des intradiegetischen Erzählens des Ballonfahrers, wird dem Ich-Erzähler bewusst, was geschehen war: das Nichtwissen von Münchhausen verwandelt sich so in Wissen - der Ablauf des Experiments offenbart sich ihm und das anfängliche Rätsel ist gelöst. Denn die Veränderungen der Perspektive in der erzählten Welt erinnern in der Tat an den Ablauf von Experimenten in der Naturforschung: das anfängliche Unwissen der Figur und das Beobachten des Objekts in großer Entfernung wird zum fokussierten Blick, welcher das Objekt ,unter die Lupe" nimmt. Dies widerspricht interessanterweise einem Befund von Beese, wonach in der Übersetzung von G.A. Bürger der experimentelle Charakter verloren gegangen sei. ${ }^{34}$ Die Analyse der Perspektivwechsel zeigt jedoch auf, dass die experimentellen Bezüge in die Tiefenstruktur des Textes übertragen wurden und auf der erzählerischen Ebene präsent bleiben. Der naturwissenschaftliche Charakter des Textes wird des Weiteren bei einer Betrachtung der zeitlichen Ebene erkennbar.

Die zeitliche Ebene lässt sich in mehrere Abschnitte unterteilen: zuerst vergeht die Zeit sehr langsam. Münchhausen bewundert die „herrlichste Aussicht“ auf Konstantinopel. Die Handlung wird sodann beschleunigt, als Münchhausen anfängt, mit dem „,runden Ding“ zu kämpfen. Anschließend kommt es zur anachronischen Erzählung des Franzosen, in welcher die Zeit gerafft wird. Und so ist unter dem Aspekt des Wissenstransfers zwischen literarischem Text und naturwissenschaftlichen Inhalten auch die Reihenfolge der einzelnen Handlungsabschnitte interessant: In diesem Falle ermöglicht die Verwendung einer Anachronie die Einführung von Aussagen einer zweiten Figur, was für eine Münchhausiade eher ungewöhnlich ist, weil der Rezipient in den meisten Lügengeschichten Münchhausens nur die Gedanken der Hauptfigur kennenlernt. Das intradiegetische Erzählen des Ballonfahrers über seine achttägige Reise mit dem Wasserstoffballon, das heißt sein Bericht über eine verhältnismäßig lange Reise, wird kurz nach der Landung gerafft (Zeitraffung) und die lange und gefährliche Ballonfahrt wird vom Reisenden nachträglich dargestellt (Analepse). Der Franzose, der zehn Minuten nach dem Start die Kontrolle über sein Fluggerät verliert, wird die ganze Zeit hindurch so schnell vom Wind angetrieben, dass er das Zeitgefühl verliert. Nur sein Heißhunger und die sich unter ihm bewegenden Seelandschaften sind ein Zeichen dafür, dass er mehrere Tage lang und mit hoher Geschwindigkeit fährt.

Mit diesen Mitteln (Analepse und Zeitraffung) wird die zukünftige Anwendung des Wasserstoffballons als schnelle Transportmöglichkeit dargestellt. Die Dynamik und Geschwindigkeit des Wasserstoffballons wird durch die Gegenüberstellung des zum Anfang ruhenden Orients (mit seinen konstruierten ${ }^{35}$ schönen Landschaften

${ }^{33}$ Bürger (1981 [1786]: 45).

${ }^{34}$ Beese (2014: 52).

35 Zum Orient als Konstruktion siehe beispielsweise die Beiträge in: Attia, Iman (Hrsg.): Orient-und IslamBilder. Interdisziplinäre Beiträge zu Orientalismus und antimuslimischen Rassismus. Unrast Verlag: Münster 2007. 
sowie dem blauem Himmel) mit der europäischen Kultur (mit ihrem sich rasant entwickelnden technischen Fortschritt) unterstützt. Paradoxerweise wird die Attraktivität des geheimnisvollen Morgenlandes durch das Auftauchen der Technik aus dem Okzident in Gestalt des Wasserstoffballons noch gesteigert. Der Orient verwandelt sich plötzlich von einer idyllisch erscheinenden - fast langweiligen Welt -, in der man nach Belustigung suchen muss, zu einem mysteriösen Ort, wo geheimnisvolle Objekte in der Luft herumfliegen. Deutlich wird an dieser Stelle der spezifisch wechselseitige Zusammenhang zwischen Raum (Orient und Okzident) und Zeit der Handlung (Raffung). Aus dieser Passage resultiert, dass die räumliche und zeitliche Achse in dieser kurzen Erzählung nicht unabhängig voneinander verlaufen, sondern miteinander verschmelzen und so zur „Raumzeit“ ${ }^{\star 36}$ werden.

Wie oben schon erwähnt, lässt sich hierbei feststellen, dass die Art und Weise wie die Handlung im diegetischen Raum dargestellt wird, dem Ablauf einer naturwissenschaftlichen Versuchsanordnung ähnelt: ein isoliertes Objekt wird zuerst von einer bestimmten Entfernung aus beobachtet. Der Abschuss entspricht einem Eingriff in die Autonomie des Gegenstandes, wobei die Gewinnung von Informationen als ein Ziel dieses Eingriffes gelten kann. Im Laufe der Handlung und infolge des Eingriffes wird das Objekt vergrößert und unter die Lupe genommen. Einzelne Faktoren und Aspekte werden variiert und isoliert. ${ }^{37}$ Der Ablauf der Handlung ist in diesem Sinne analogisch zum Ablauf eines Experiments. Hingegen wird die Ballonfahrt als solche im Text nicht als Experiment im eigentlichen Sinne beschrieben: die Ballonfahrt diente keiner Gewinnung von Erkenntnissen, vielmehr folgte sie einer volksbelustigenden Absicht. Mit dem Reißen des Ventilfadens nähert sich die Erzählung wieder einem wissenschaftlichen Experiment an, welches jedoch außer Kontrolle gerät. An dieser Stelle sei an Joseph Vogls ${ }^{38}$ Überlegungen zu seinen Poetologien des Wissens erinnert, wonach Wissen überall präsent sei - nicht nur in einem wissenschaftlichen Experiment, sondern ebenso in Gesetzesentwürfen und Verordnungen, in sprachlichen Wendungen oder eben auch in einem literarischen Text. Das Wissen zur Aerostatik taucht also dort auf, wo man es am wenigsten erwartet - in einem literarischen Text, der heutzutage als Kindergeschichte gilt.

${ }^{36}$ An dieser Stelle schöpfe ich nicht nur aus dem literaturwissenschaftlichen Werkzeugkasten Bachtins (siehe Bachtins Formen der Zeit und des Chronotopos im Roman, in der neusten deutschen Ausgabe unter dem Titel Chronotopos laufend. Suhrkamp: Frankfurt am Main [1975] 2008), sondern auch - ähnlich wie er - aus der Physik - insbesondere der Relativitätstheorie Einsteins (siehe zudem Minkowskis Begriff der Raumzeit: „Die dreidimensionale Geometrie wird ein Kapitel der vierdimensionalen Physik. Raum und Zeit sollen zu Schatten herabsinken und nur eine Welt an sich bestehen, Minkowski (1911: 103)).

37 Griesecke/Kogge (2005: 45).

38 Vogl (1999: 11). 


\section{Kritik des Abendlandes und wissenschaftlicher Bericht}

Mit dem Wissen über das Objekt ändert sich das Wissen über die eigene Welt: laut Polaschegg dient die Übertragung der Handlung aus Europa in den Orient in literarischen Texten des 18. Jahrhunderts häufig einer Selbstreflexion - einem Nachdenken über die Ereignisse und Prozesse, welche die hiesige Wirklichkeit gestalten. ${ }^{39}$ So wird mit der Platzierung der Geschichte des Vierten Seeabenteuers in dem Nahen Osten die Debatte über die technischen Errungenschaften des Okzidents aufgenommen. In der Münchhausenerzählung entpuppt sich hierbei die neueste Technik der eigenen Welt als bloßer Schein - als Enttäuschung sowie als nutzloses „Kunststück ${ }^{\text {“40 }}$, weil es nicht einmal gelingt, den Ballon richtig zu steuern. Die neuesten Errungenschaften der abendländischen Technik versagen und nur dank der Intuition und des zufälligen Anstoßes Münchhausens wird der Ballonreisende aus seiner technischen Falle befreit. Stabilität und Sicherheit der Technik werden in Frage gestellt: zuerst zerfällt das Ventil und bei der Landung schließlich wird der Wasserstoffballon „,im Herabfallen vollends ganz und gar zu Stücken zerrissen“ “41 die „Entdeckung, welche in diesem Jahrhundert wahre Epoche macht“42 entpuppt sich so als bloßer mit Luft und erhitzten Dämpfen ${ }^{43}$ gefüllter Stoff, dessen Nutzen auf das „,wissenschaftliche Spektakel“44 und die ,herrliche Scene“45 reduziert wird.

Nicht nur ihre Technik, sondern die ganze europäische Gesellschaft, ${ }^{46}$ wird in Gestalt der Figur des Ballonfahrers beanstandet. Beese geht davon aus, dass der französische Ballonfahrer in Bürgers Münchhausen den Luftschifffahrtspionier Jean-Pierre Blanchard ${ }^{47}$ repräsentieren soll. ${ }^{48}$ Sie macht diesbezüglich auf

39 Polaschegg (2008: 28).

40 Bürger (1981 [1786]: 45).

${ }^{41}$ Ebd.

42 Pingeron (1784: 31$)$.

${ }^{43}$ Lichtenberg: Göttingische Anzeigen von gelehrten Sachen, 1. Band, 7 Stück, 1784, S. 57-72.

${ }^{44}$ Ebd.

45 Cavallo (1786: 119).

46 Beese (2014: 48).

47 Jean-Pierre Blanchard (1753-1809) war ein französischer Ballonfahrer. Nach seinen fehlgeschlagenen ersten Flugversuchen mit einem - mit Rudern und Flügeln ausgestatteten - Luftschiff nutzte er schließlich den von Jacques Alexandre César Charles (1746-1823) erfundenen Wasserstoffballon, um in ganz Europa spektakuläre Ballonflugversuche aufzuführen. Viele zeitgenössische Gelehrte kritisierten ihn dafür als ideenlosen Laien, der seine Ballonshow unter andrem mit Tieren durchführte (die er mit einem Fallschirm aus dem Ballon abwarf), nur um sich bereichern zu können (siehe beispielsweise die Beschreibung seiner Flugreise in England von Tiberius Cavallo (1786: S. 116-121). Positiver berichtete der Breslauer Professor Anton Gottfried Steiner über Blanchard, der ihn persönlich kennenlernte. Auch Blanchard beschrieb seine Fahrten in Zeitungen und Berichten. Als Beispiel kann der folgende schwer zugängliche Text dienen: Analyse de la Machine aërostatique que j'ai inventée (sic!-a.w.) et exécutée a Varsovie pendant l'année 1789 et 1790. Für die Gewinnung des Textes danke ich an dieser Stelle Stanisław Siess-Krzyszkowski am Centrum Badawcze Bibliografii Polskiej Estreicherów (Uniwersytet Jagielloński).

48 Beese (2014: 39-40). 
intertextuelle Verweise auf zeitgenössische Zeitschriften aufmerksam, welche die Person Blanchards beschreiben und aus deren Darstellungen man schlussfolgern kann, dass die Figur im Text bestimmte charakteristische Eigenschaften JeanPierre Blanchards aufweist. An dieser Stelle könnte man geneigt sein, die Einführung der Figur des Franzosen unter politischen Aspekten zu betrachten: der adelige Deutsche schießt auf den „abgehobenen“ bürgerlichen Franzosen. Diese Differenz wird jedoch im Text dekonstruiert: dem Franzosen, der ,dem menschlichen Geschlechte außerordentlich wichtige Dienste geleistet ha[t]“ ${ }^{49}$ gelingt es nicht seine Haltung zu wahren, denn ihm ist übel. Zudem repräsentiert die Figur Münchhausens auch keinen typischen Adeligen, sie ist vielmehr eine Projektion des abenteuerlichen Lebens.

Beese macht ebenfalls sichtbar, dass in der Ausgabe Bürgers die ,demonstrative Verschwendung ${ }^{“ 50}$ der westlichen Gesellschaft vorgeführt wird. Der Ballon wird als Kunst für „Nachgaffer“ ${ }^{\text {“51 }}$ dargestellt und das Lammfleisch wird verschwenderisch ins Meer geschmissen. Der stolze Franzose mit seinen Medaillen und der fehlenden Kreativität zum Bau einer eigenen Maschine zielt nur auf die Anerkennung und den Lob der Anderen ab - doch selbst das mag nicht gelingen: denn der Ich-Erzähler nimmt in der Erzählung die Rolle des ,Pöbels‘ ein, welcher den damaligen Ballonvorstellungen üblicherweise beiwohnte. Der „Theaterkünstler" schafft es jedoch nicht, vor seinem Publikum zu punkten: denn schon bald nach dem Aufstieg scheitert das Spektakel und der Ballon treibt davon. Zum Ende der Reise stellt Münchhausen schließlich das extrem zusammengeschrumpfte Publikum für die „Ballonshow“ Blanchards dar - die anfänglichen Schaulustigen sind schon längst nach Hause gegangen. Diese literarische Abfertigung wird durch die parodiehafte Transformierung von wissenschaftlichen Berichten zur Ballonfahrt noch verstärkt.

Der Franzose ist der europäische „Showman“, welcher den Ballon zwar nicht erfunden hat, ihn aber vorführt und der in der Erzählung das Wissen über den Wasserstoffballon an den Laien Münchhausen vermittelt. Die Kommunikation dieses Wissens weist hierbei Merkmale von zeitgenössischen Briefen und Berichten ${ }^{52}$ von Wissenschaftlern um 1800 auf sowie von Blanchards eigenen Berichten..$^{53}$ Die Aussagen Blanchards erinnern - wie Beese ebenfalls bemerkt ${ }^{54}$ - an die Briefe der Pioniere der Ballonfahrt, insbesondere was den Aufbau betrifft. So versucht der Franzose - wie in zeitgenössischen Berichten üblich - zum Anfang zu erklären,

49 Bürger (1981 [1786]: 45).

50 Siehe dazu: Beese (2014: 54).

51 Bürger (1981 [1786]: 45).

52 Beese geht jedoch davon aus, dass nur bei der Ausgabe von Raspe eine Ähnlichkeit mit wissenschaftlichen Berichten nachgewiesen werden könne. Mir geht es darum zu zeigen, dass dies auch bei Bürgers Ausgabe der Fall ist. Vgl. Beese (2014: 49).

53 Vgl. ebd.

${ }^{54}$ Vgl. ebd, S. 39. 
von wem die Idee zur Luftfahrt stamme und wer den Ballon erstmalig konstruierte. Er selbst war daran unbeteiligt, er hat die Erfindung nur für seine Zwecke ,geliehen". Geschickt wird hier die Frage nach den Erfindern der Ballonfahrt umgangen - weder die Namen der Brüder Montgolfier noch der von Charles ${ }^{55}$ tauchen auf. In den Texten von Bürger und von Raspe wird dadurch gleichermaßen eine Positionierung im Konflikt zwischen den beiden Erfinderlagern vermieden.

Es entspricht ebenfalls den zeitgenössischen Berichten über die Ballonfahrt, dass der Franzose von seinem eigenen Flugversuch erzählt und berichtet, wie er sich in die Luft erhebt und der Pöbel mit Begeisterung seinen Aufstieg beobachtet. Nicht nur räumlich, sondern auch mit dem Satz: ,um von oben herab vor den Augen vieler tausend Nachgaffer Kunststücke damit zu machen “56, distanziert er sich strikt von den „Nachgaffern“. ${ }^{57}$ Ähnlich wie in vielen wissenschaftlichen Berichten der Zeit ist das Ziel seiner Fahrt hierbei klar definiert: er bricht von Cornwall auf und möchte nach Exeter fahren. Der Erzählende versucht gegenüber Münchhausen seine Flughöhe zu bestimmen, da er aber an Bord über keine wissenschaftlichen Geräte verfügt, denn es ging ihm ja lediglich um die Aufführung eines „Kunststücks“"58, umschreibt er die Höhe ungenau und floskelhaft als „unermesslich". 59

Die Versuche, die eigene Flughöhe zu bestimmen, das klar definierte Reiseziel sowie die beschriebenen physiologischen Unannehmlichkeiten (Übelkeit) ${ }^{60}$ weisen Ähnlichkeit mit zeitgenössischen Berichten Blanchards auf, die durch die literarische Darstellung parodiert ${ }^{61}$ und als übertreibend dargestellt wurden. An dieser Stelle lässt sich gut beobachten, wie die Literatur das Wissen zur Aerostatik transformierte: ursprünglich schriftliche Beschreibungen, hier also die Berichte über naturwissenschaftliche Experimente mit Wasserstoff- und Heißluftballons, werden aus den zeitgenössischen Lehrbüchern, wissenschaftlichen Briefwechseln sowie Zeitungsartikeln in die Aussagen des Franzosen umgewandelt. Die Rede des Ballonfahrers gliedert sich hierbei in zwei längere Monologe. Der Ich-Erzähler unterbricht ihn, um dem Ballonfahrer zu erklären, wo er sich befindet. Der

${ }^{55}$ Um 1800 lagen die Anhänger des (von den Brüdern Montgolfier erfundenen) Heißluftballons im Streit mit den Anhängern des (von Jaques Alexandre César sowie den Gebrüdern Robert erdachten) Wasserstoffballons. Im deutschsprachigen Raum berichtet Martin Wieland in seinen zwei Texten Die Aeropetomanie und Die Aeronauten über diesen Disput.

56 Bürger (1981 [1786]: 45).

57 Ebd.

58 Bürger (1981 [1786]: 45).

59 Ebd.

${ }^{60}$ Auf „eine kleine Übelkeit“ wegen des Geruchs des Wasserstoffs bei seinem Gefährten verweist Jungius in seinem Bericht an das Publikum über meine Luftreise am 19ten May 1806. Interessant ist jedoch, dass man sich in zeitgenössischen Lehrbüchern und Berichten am häufigsten über die Kälte während eines Ballonflugs sowie über verstopfte Ohren beschwerte und nicht über Übelkeit. Z.B. Cavallo (1786: 73), Murr (1784: 106).

${ }^{61}$ Beese (2014: 41). 
Luftschiffer vermutet vage, dass er sich nicht mehr in der Nähe von Cornwall aufhalte. Münchhausen macht ihm aber bewusst, dass seine Flugversuche stärker fehlgegangen sind, als es der Luftfahrer auch nur ahnte: sein Ballon hat den Raum des Okzidents verlassen und die Grenzen zur Welt des Orients überschritten. Der Mensch des späten 18. und frühen 19. Jahrhunderts - hier in der Figur des Franzosen - überquert nicht nur die vertikale Grenze des Raumes, was ihm mit der Ballonfahrt erstmals möglich wurde. Mit Hilfe der Technik stellt er auch die alten politischen Konstruktionen, wie insbesondere die Trennungslinie zwischen Orient und Okzident infrage. Der Ballonfahrer bemerkt hierbei nicht einmal, dass er die Grenzen „seiner“ Welt überschritten hat. Diese Grenzziehung wird erst durch seine zweiteilige Rede wieder hergestellt und betont. Der erste Teil seiner Rede symbolisiert hierbei das Nichtwissen. Denn erst im zweiten Teil wird klar, wie es zu dieser Situation kam (der Ventilfaden zerriss). Beide Teile haben die Form eines Monologs, die Länge der Rede symbolisiert hierbei auch die Länge der Dauer der Ballonfahrt.

Durch die deutliche Charakterisierung der Welt des Okzidents, in der Münchhausen auf Tiere und Ballons schießt, als kalt, technisch und rücksichtslos wird der Unterschied zur Wärme des sonnigen Morgenlandes akzentuiert. Hierbei wird erstens der brutale Umgang mit Tieren deutlich, die im Abendland gezwungen sind, an den Flugexperimenten der Menschen teilzunehmen oder auch einfach nur zu deren Belustigung aus großen Höhen mit dem Fallschirm abgeworfen werden. Obwohl die Fahrt des Franzosen ursprünglich nicht als Experiment geplant war, beinhaltet sie daher nicht nur die Elemente eines „Kunststückes“, sondern auch Eigenschaften eines Tierexperiments und zwar gerade von dem Zeitpunkt an, ab welchem das Kunststück schief geht. Die Ballonfahrt mit dem Tier an Bord misslingt: anstatt jedoch menschlichen Erfindergeist zu beweisen und dennoch sicher auf dem Boden zu landen, wird das Tier geschlachtet und sein Kadaver in der Sonne gebraten. Das Tier ist hier nur Objekt, also „Versuchsmaterial“. ${ }^{62}$ Mit diesem kann man sich zum einen ernähren, sobald das eigene Leben gefährdet ist.

Des Weiteren wird am Beispiel des Schafs deutlich, wie in der Münchhausiade das unlogische Moment die literarische Fiktion ankurbelt. Außer den Tatsachen, dass um 1800 längere Fahrten mit dem Ballon als unmöglich galten ${ }^{63}$ und

62 Borgards (2010: 346-347).

${ }^{63}$ Noch im Jahre 1786 galt eine mehrtägige Fahrt mit einem Wasserstoff- oder Heißluftballon als empirisch unmöglich - vor allem deswegen, weil die warme Luft oder der Wasserstoff relativ schnell aus den damals gebräuchlichen porösen Stoffhüllen entwichen. Als Beispiel für eine fehlgeschlagene Fahrt, die mehrere Tage dauern sollte, kann ein Flugversuch angeführt werden, der am 19. Januar 1784 in Lyon stattfand. Diese Reise war die erste Reise von Joseph-Michel Montgolfier. Der Miterfinder des Heißluftballons hatte geplant zusammen mit anderen Teilnehmern von Lyon aus nach Paris zu fliegen, was mehrere Tage in Anspruch genommen hätte. Die Ballonhaut der „,unglücklichen Maschine" bestand aus Leinwand und Papier und konnte die Apparatur nicht lange in der Luft halten. Schlechtes Wetter setzte dem Ballon zu - Nässe und Schnee zersetzten die Ballonhülle und führten schließlich dazu, dass die Teilnehmer ihre Pläne verwarfen und noch am gleichen Tag lande- 
dass sich ein Ballon nicht so leicht mit einer Vogelflinte abschießen lässt, dient auch das Braten des Schafes in der Sonne als ein solches unlogisches Moment der Erzählung. Des Weiteren öffnet das Faktum, dass der Rezipient der Erzählung nicht genau weiß, welche „Kunstücke“ der Franzose noch mit dem Schaf durchführen wollte, Raum für diesbezügliche Spekulationen.

Drittens wird das Schaf zum Mittel der (tier-)experimentellen Erkenntnis: das Tier wird zum Stellvertreter des menschlichen Versuchsobjekts, an dem beobachtet werden kann, was vermeintlich passiert, wenn ein Lebewesen in großer Höhe der direkten Einwirkung der Sonnenstrahlen ausgesetzt ist. Und zum vierten schließlich zeigt das sterbende Schaf, welche negativen Konsequenzen ein schiefgegangenes Experiment mit dem Wasserstoffballon für die Physiologie des Menschen haben kann. Dies entspricht den Überlegungen von Roland Borgards, der aufzeigt, ${ }^{64}$ dass die Menschen im späten 18. Jahrhundert erstmals anfingen das Tier als „Sender“ zu betrachten. Das, was dem Tier während eines Experiments zustieß, galt seitdem als nur teilweise auf die Pathologie des Menschen (des „Empfängers") übertragbar. ${ }^{65}$ Noch im 17. und bis ins frühe 18. Jahrhundert hinein wurde das Tier hingegen als direkter Stellvertreter ${ }^{66}$ des Menschen betrachtet. Erst seit dem späten 18. Jahrhundert gilt, dass die Erkenntnisse, die aus Tierexperimenten gewonnen werden, nur mittelbar auf den Menschen verwendbar sind. ${ }^{67}$ Die quasiexperimentellen Erfahrungen mit dem Schaf sind also nur bedingt auf die menschliche Physiologie übertragbar - das Schaf ist hierbei ein Sender, der Informationen darüber vermittelt, was mit dem menschlichen Körper unter den Umständen geschehen könnte.

\section{Das Gedankenexperiment als Mittler zwischen Wissenschaft und Literatur}

Bachmann-Medick bezeichnet die Lügengeschichten Münchhausens als ein „Darstellungsmedium von Erkenntnisunsicherheiten“" ${ }^{68}$ das wieder den Begriff des Experiments in Erinnerung ruft. ${ }^{69}$ Und auch Beese kommt in ihrer Interpretation zu

ten - nicht in Paris, sondern auf einer Wiese in der Nähe von Lyon. Ausführliches zur Berichterstattung über den Lyoner Versuch seitens der deutschen Rezeption siehe: Cavallo (1786: 79), für die polnische Rezeption siehe: Gazeta Warszawska 1784, Nr. 9, S. 6, Nr. 10, S. 7, Nr. 11, S. 4, Nr. 12, S. 2, 3, 7 .

64 Borgards (2010: 349-352).

${ }^{65}$ Im Gegensatz zum 17. und frühen 18. Jahrhunderts, als das Tier noch als Stellvertreter des Menschen betrachtet wurde (so genannte Repräsentation: Borgards 2010). Mehr zur Repräsentation und zur Übertragung findet sich bei: Borgards (2010: 349-352).

66 Vgl. Borgards (2010: 351).

67 Vgl. ebd.

${ }^{68}$ Bachman-Medick (1997: 54).

${ }^{69}$ Ebd. 
dem Schluss, dass die Flugepisode in der Fassung Raspes als ein Gedankenexperiment bezeichnet werden könnte, ${ }^{70}$ da sie sich mit ihrem Spielwitz an der Grenze zwischen „Satire und Vision“"71 befinde. Dies führt zu der Frage, ob auch die deutschsprachige Version von den Abenteuern Münchhausens (und insbesondere das Vierte Seeabenteuer) als Form des Gedankenexperiments betrachtet werden kann.

Der Begriff des Gedankenexperiments wurde gegen Ende des 19. Jahrhunderts von seinem geistigen Vater Ernst Mach (1838-1916) geprägt. ${ }^{72}$ Der Wissenschaftstheoretiker definiert diese Form des Experimentierens ${ }^{73}$ als das Sammeln von Beobachtungen und Erfahrungen. ${ }^{74}$ In dieser weiten Definition ist in gewisser Weise der Unterschied zwischen dem empirischen und dem gedanklichen Experiment aufgehoben. Denn Mach geht davon aus, dass es ,eine rein experimentelle Forschung [...] übrigens nicht [gibt] “" ${ }^{75}$ da jedem empirischen Experiment immer ein gedankliches Experiment vorausgehen müsse. Man solle daher sowohl das Gedankenexperiment als auch das empirische Experiment als eine „Methode der Variation“76 oder „Methode der Veränderung“" verstehen, wobei sich jedoch das Gedankenexperiment ,auf höherer intellektueller Stufe“77 abspiele. An diese relativ breite Definition knüpft auch Thomas Kuhn an, dem das Gedankenexperiment als der Motor der Wissenschaft gilt. ${ }^{78}$ Als solcher erfülle es ihm zufolge zum einen

${ }^{70}$ Beese (2014: 179).

${ }^{71}$ Ebd.

72 Einen theoriegeschichtlichen und historischen Überblick über Gedankenexperimente liefert Krauthausen (2010). Die Autorin (2010: 278) betont die lange Geschichte des Gedankenexperiments, welche bis in die Antike zurückreicht. Zum Gedankenexperiment siehe auch den Sammelband Science \& Fiction. Über Gedankenexperimente in Wissenschaft, Philosophie und Literatur von Macho und Wunschel (Hrsg.) aus dem Jahre 2004.

73 Mach (1917: 143). Dieses Verfahren sei nicht nur für wissenschaftliches Handeln charakteristisch. Vielmehr handele es sich dabei um eine Eigenschaft, die allen Menschen (und Tieren) angeboren sei und die ihren Ursprung in der Neugierde habe. Mach schreibt: „Wenn wir beobachten, wie ein Kind, welches die erste Stufe der Selbständigkeit erreicht hat, die Empfindlichkeit seiner eigenen Glieder prüft, wie es von seinem Spiegelbilde, oder von seinem eigenen Schatten im hellen Sonnenschein befremdet, durch Bewegungen die Bedingungen desselben zu ermitteln sucht, wie es sich im Werfen nach einem Ziele übt; so müssen wir sagen, daß die instinktive Neigung zum Experimentieren dem Menschen angeboren ist."

${ }^{74}$ Wie Krauthausen bemerkt, wird der Begriff des Gedankenexperiments in verschiedenen Epochen anders verstanden: „Paradoxa, Rätsel, mathematische Intuitionen, geometrische Konstruktionen, fiktive Experimentalapparate, fingierte/imaginäre (d.h. realisierbare, aber nicht durchgeführte) Experimente, nicht realisierbare Experimente, absurde Experimente, von kontrafaktischen Annahmen ausgehende Explorationen, Experimentalsituationen mit explizit phantastischen Entitäten“ (Krauthausen, S. 305). Damit wird die Tatsache verbunden, dass das Gedankenexperiment oft als „Fiktion an sich" betrachtet wurde (Ebd. S. 318).

75 Mach (1987 [1923]: 305).

76 Mach (1917: 143).

77 Mach (1917: 146).

${ }^{78}$ Vgl. Krauthausen (2010: 286) sowie einen Aufsatz von Thomas Kuhn: Eine Funktion für das Gedankenexperiment, in: Die Entstehung des Neuen, Frankfurt am Main: Suhrkamp 1992, S. 327-356. 
eine epistemische Funktion, da es dazu befähige, wissenschaftliche Anomalien zu antizipieren, die sich zu wissenschaftlichen Krisen $^{79}$ auswachsen können. Zweitens ermögliche das Gedankenexperiment einen Perspektivenwechsel, ${ }^{80}$ durch welchen das, was früher nicht bemerkbar war oder anders gesehen wurde, in ein ganz anderes Licht gestellt werden kann. Wie oben schon erwähnt, wird dieser Perspektivenwechsel in der Literatur häufig durch die Vergrößerung oder durch die Verkleinerung des betreffenden Objekts erreicht. ${ }^{81}$

Beispielsweise wird die Perspektive im Vierten Seeabenteuer durch die Techniken der Verkleinerung (die Beobachtung eines weit entfernten Ballons) und der Vergrößerung (die Landung des Franzosen in einem „niedlichen Wagen“ vor den Füßen des Lügenbarons) gewechselt. Dies dient nicht lediglich der Darstellung der hohen Geschwindigkeit, welche der Ballon bei seiner Reise aufnimmt, das heißt der Darlegung der technischen Errungenschaft, die das Fluggerät als Verkehrsmittel repräsentiert. Das Beibehalten des Perspektivenwechsels in der deutschen Fassung weist vielmehr Züge des Gedankenexperiments auf und macht deutlich, wie die Spuren der Naturforschung in der deutschsprachigen Version des Münchhausens weiter enthalten blieben.

Ein zweiter Aspekt, der in Bürgers Münchhausenversion an ein Gedankenexperiment erinnert, ist ebenfalls mit den Arbeiten Thomas Kuhns verbunden: laut ihm dient das Gedankenexperiment als ein „hochsensibles Instrument", welches eine Krise des wissenschaftlichen Wissens antizipiert. ${ }^{82}$ Dementsprechend geht beispielsweise auch Blättler davon aus, dass jedes Experiment neben einer explorativen Funktion immer auch über demonstrative Momente verfüge. ${ }^{83}$ Die demonstrative Funktion des Experiments ermögliche es hierbei, eine bestimmte Wirklichkeit zu bestätigen. ${ }^{84}$ In diesem Sinne lässt sich festhalten, dass das Gedankenexperiment in der Münchhausenerzählung die aerostatische Krise der Montgolfière , bestätigt' ${ }^{\star}$. Hierbei wird ihre historische Verdrängung durch die Charlière mitaufgezeigt, wenngleich diese Vorgänge im Text nicht direkt angesprochen werden. Die Verwendung der Charlière im Text weist darauf hin, dass im Jahre 1786

${ }^{79}$ Zum Begriff der Krise, siehe insbesondere das Kapitel VII: Krisen und das Auftauchen wissenschaftlicher Theorien bei Kuhn (1973). Nach der Darstellung der drei historischen Beispiele für Paradigmenwechsel (die kopernikanische Revolution, die Ablösung der Phlogiston- durch die Oxidationstheorie sowie die Verdrängung der Äthertheorie durch die Relativitätstheorie) wird auf den Seiten 87 und 88 kurz der Begriff der wissenschaftlichen Krise definiert.

${ }^{80}$ Krauthausen (2010: 288).

${ }^{81}$ Auf dieses Verfahren mit/in Gedanken zu experimentieren, verweisen unabhängig voneinander sowohl Albrecht Schöne als auch Gerhard Neumann in ihren Überlegungen zur Aphoristik bei Georg Christoph Lichtenberg (1742-1799). Siehe: Schöne (1983) sowie Neumann (1976).

82 Krauthausen (2010: 286)

${ }^{83}$ Blättler (2010: 238).

${ }^{84}$ Ebd. 
die Montgolfière nur selten eingesetzt wurde. ${ }^{85}$ Es lässt sich daraus schlussfolgern, dass das Gedankenexperiment in literarischen Texten die wissenschaftlichen Krisen (z.B. in der Aerostatik) nicht nur antizipieren kann, sondern sie auch auf die literarische Ebene hebt und so allgemein und öffentlich macht.

Drittens wird das Gedankenexperiment auch als Hilfsmittel verwendet, um vorherzusagen, was passieren kann, wenn das technische Gerät versagt und nicht mehr kontrollierbar - hier: nicht mehr lenkbar - ist. Trotz der Tatsache, dass die Handlung viele Paradoxa und wissenschaftliche Ungenauigkeiten enthält, ${ }^{86}$ beinhaltet die Geschichte daher ein Gedankenexperiment, insofern sie entwirft, was in einer solch absurden Situation geschehen könnte. Laut Mach ist das Gedankenexperiment seinem Wesen nach eng mit dem Paradoxon verbunden, da beide Lernprozesse unterstützen sowie hinterfragen und dadurch weiteres Denken anregen:

Nicht nur lernt man durch das Paradoxe am besten die Natur eines Problems fühlen, welches ja eben durch den paradoxen Gehalt zu einem Problem wird, sondern die widerstreitenden Elemente lassen auch die Gedanken nicht mehr zur Ruhe kommen, und lösen eben den Prozeß aus, den wir als Gedankenexperiment bezeichnet haben. ${ }^{87}$

Viertens gibt das Gedankenexperiment dem Rezipienten die Möglichkeit, am eigentlich ,undemokratischen' Projekt der Ballonfahrt zu partizipieren. So konnten die meisten Menschen um 1800 die Ballons lediglich von unten beobachten, die Teilnahme an den Flügen beschränkte sich anfänglich auf den sehr kleinen und elitären Kreis von Erfindern. Die Verwobenheit aus Gedankenexperiment und Münchhausiade eröffnete daher einen Weg, die Ballonfahrten in gewisser Weise zu demokratisieren. Dank der Gedankenexperimente in der volkstümlichen Münchhausiade konnten alle Rezipienten am Flug teilnehmen, indem sie ihn mithilfe des literarischen Textes nacherlebten. Den literarischen Texten kam also in diesem Demokratisierungsprozess der frühen Luftfahrt (zusammen mit den anderen Medien der Öffentlichkeit - z.B. wurden Berichte über die aerostatischen Fahrten in der Presse veröffentlicht) eine wichtige Funktion zu: die Literatur eröffnete einen Weg zur späteren vollen Demokratisierung des Fliegens, wie sie sich im späten 18. Jahrhundert abspielte. ${ }^{88}$

85 Über das Heißluftballonverbot berichtete beispielsweise Lichtenberg in seiner schriftlichen Korrespondenz (siehe insbesondere seinen Brief an Franz Ferdinand Wolff vom 22. März 1784 (Schriften und Briefe, S. 838) sowie im Brief an Georg August Ebell vom 23. März 1784 (Schriften und Briefe, S. 838-839)). Für den polnischsprachigen Raum finden sich entsprechende Berichte in der Gazeta Warszawska 1784, Nr. 32 und Nr. 41. Zudem findet sich in der Nr. 43 eine Bemerkung darüber, dass in Genua das Fliegen mit Heißluftballons verboten wurde. Bei Zuwiderhandlung wurde eine Geldstrafe in Höhe von 25 Talern verhängt.

${ }^{86}$ So müsste zum Beispiel bei einem zerrissenen Ventilfaden allmählich die Luft aus dem Ballon entweichen, so dass dieser auf keinen Fall 3000 Kilometer weit reisen könnte.

87 Mach (1917: 153).

88 Über die Demokratisierung der Luftfahrt schreibt G. Ulrich Großmann im Vorwort zum Katalog der Ausstellung im Germanischen Nationalmuseum vom 20. November bis 22. Februar 2015 in Nürnberg: Von oben gesehen. Die Vogelperspektive. Nürnberg: Verlag des Germanischen Nationalmuseum 2014. 


\section{Literatur}

\section{Primärliteratur}

Bürger, Gottfried August: Wunderbare Reisen zu Wasser und Lande, Feldzüge und lustige Abenteuer des Freiherrn von Münchhausen: wie er dieselben bei der Flasche im Zirkel seiner Freunde selbst zu erzählen pflegt. Lepizig 1981 [1786].

\section{Zeitgenössische Quellen}

Brockhaus Conversations-Lexikon Bd. 3. Amsterdam 1809, S. 194-195.

Brockhaus' Kleines Konversations-Lexikon, fünfte Auflage, Band 2. Leipzig 1911, S. 225.

Cavallo, Tiberius: Geschichte und Praxis der Aerostatik. Leipzig 1786.

Faujas de Saint-Fond, Barthélemy: Beschreibung der Versuche mit den aerostatischen Maschinen. Leipzig 1784.

Gazeta Warszawska 1784, Nr. 9, S. 6, Nr. 10, S. 7, Nr. 11, S. 4, Nr. 12, S. 2, 3, 7, Nr. 31, Nr. 41.

Göttingische Anzeigen von gelehrten Sachen, 1. Band, 7 Stück, 1784, S. 57-72.

Jungius, Friedrich Wilhelm: Bericht an das Publikum über meine Luftreise am 19ten May 1806. Berlin 1806.

Lichtberg, Georg Christoph: Schriften und Briefe [Herausgegeben von Wolfgang Promies]. München 1967.

Murr C.G. von: Der Herren Stephan und Joseph von Montgolfier Versuche mit der von ihnen erfundenen aerostatischen Maschine. Nürnberg 1784.

Pingeron, Jean Claude: Kunst, den Luftball nach jenen des Herrn von Montgolfier zu verfertigen. o.O. 1784.

Steiner, Anton Gottfried: Geschichte der zu Breslau den 27. Mai von Herrn Blanchard unternommenen Luftreise. Breslau 1789. Der Bericht wurde von Peter P.E. Günther im Jahre 1989 veröffentlicht: Zeitgenössische Berichte der ersten bemannten Luftfahrt in Breslau. Berlin 1989.

Wieland, Martin: Die Aeronauten. In: Sämtliche Werke [Herausgegeben von J.G. Gruber]. Leipzig 1826, S. 349-431.

Wieland, Martin: Die Aeropetomanie. In: Sämtliche Werke [Herausgegeben von J.G. Gruber]. Leipzig 1826, S. 315-346.

\section{Forschungsliteratur}

Attia, Iman (Hrsg.): Orient-und IslamBilder. Interdisziplinäre Beiträge zu Orientalismus und antimuslimischen Rassismus. Münster 2007.

Bachmann-Medick, Doris: Fremddarstellung und Lüge: Übersetzung als kulturelle Übertreibung am Beispiel von Münchhausens Lügengeschichten. In: Bachmann-Medick, Doris (Hrsg.): Übersetzung als Repräsentation fremder Kulturen. Berlin 1997, S. 42-68.

Bachtin, Michail: Formen der Zeit und des Chronotopos im Roman, in der deutschen neusten Ausgabe unter dem Titel: Chronotopos. Frankfurt am Main [1975] 2008.

Beese, Melanie: Münchhausens wunderbare wissenschaftliche Abenteuer zu Wasser und in der Luft und wie er diese zu erzählen pflegt. Eine literatur- und wissensgeschichtliche Studie. Bielefeld 2014.

Germanica Wratislaviensia 140, 2016

(C) for this edition by CNS 
Blättler, Christine: Demonstration und Exploration. Aspekte der Darstellung im wissenschaftlichen und literarischen Experiment. In: Gamper, Michael (Hrsg.): Experiment und Literatur. Göttingen 2010, S. 236-251.

Borgards, Roland: Das Tierexperiment in Literatur und Wissenschaft. In: Gamper, Michael (Hrsg.): Experiment und Literatur. Göttingen 2010, S. 345-360.

Doosry, Yasmin (Hrsg.): Von oben gesehen - die Vogelperspektive: Ausstellung im Germanischen Nationalmuseum, Nürnberg, vom 20. November 2014 bis 22. Februar 2015. Nürnberg 2014.

Griesecke, Birgit / Kogge, Werner: Was ist eigentlich ein Gedankenexperiment? Mach, Wittgenstein und der neue Experimentalismus. In: Krause, Marcus / Pethes, Nicolas: Literarische Experimentalkulturen: Poetologien des Experiments im 19. Jahrhundert. Würzburg 2005, S. 41-72.

Krauthausen, Karin: Wirkliche Fiktionen. Gedankenexperimente in Wissenschaft und Literatur. In: Gamper, Michael (Hrsg.): Experiment und Literatur. Göttingen 2010, S. 278-320.

Kuhn, Thomas: Eine Funktion für das Gedankenexperiment. In: Die Entstehung des Neuen, Frankfurt am Main 1992, S. 327-356.

Link, Jürgen: "Einfluss des Fliegens! Auf den Stil selbst!” Diskursanalyse des Ballonssymbols. Diskursanalyse des Ballonsymbols. In: Link, Jürgen: Bewegung und Stillstand in Metaphern und Mythen. Fallstudien zum Verhältnis von elementarem Wissen u. Literatur im 19. Jahrhundert. Stuttgart 1984, S. 151-152.

Link, Jürgen: Literaturanalyse als Interdiskursanalyse. Am Beispiel des Ursprungs literarischer Symbolik in der Kollektivsymbolik. In: Fohrmann, Jürgen / Müller, Harro (Hrsg.): Diskurstheorien und Literaturwissenschaft. Frankfurt am Main 1988, S. 284-306.

Mach, Ernst: Erkenntnis und Irrtum. Skizzen zur Psychologie der Forschung. Berlin [1917] 2014.

Mach, Ernst: Populär-wissenschaftliche Vorlesungen. Wien 1987.

Macho, Thomas / Wunschel, Annette: Science \& Fiction. Über Gedankenexperimente in Wissenschaft, Philosophie und Literatur. Frankfurt am Main 2004.

Martínez, Matias / Scheffel, Michael: Einführung in die Erzähltheorie. München 2009.

Minkowski, Hermann: Raum und Zeit. In: Hilbert, David: Gesammelte Abhandlungen von Hermann Minkowski. Leipzig und Berlin 1911.

Neumann, Gerhard: Ideenparadiese. Untersuchungen zur Aphoristik von Lichtenberg, Novalis, Friedrich Schlegel und Goethe. München 1976.

Pethes, Nicolas: Literatur- und Wissenschaftsgeschichte. Ein Forschungsbericht. In: IASL 28, 2003, S. 181-231.

Pethes, Nicolas: Poetik/Wissen. Konzeptionen eines problematischen Transfers. In: Brandstetter, Gabriele / Neumann, Gerhard: Romantische Wissenspoetik. Die Künste und die Wissenschaften um 1800. Würzburg: 2004, S. 341-372.

Polaschegg, Andrea: Die Regeln der Imagination. Faszinationsgeschichte des deutschen Orientalismus zwischen 1770 und 1850. In: Goer, Charis / Hofmann, Michael: Der Deutsche Morgenland. Bilder des Orients in der deutschen Literatur und Kultur von 1770 bis 1850 . München 2008, S. 13-36.

Schöne, Albrecht: Aufklärung aus dem Geist der Experimentalphysik. Lichtenbergsche Konjunktive. München 1983.

Vogel, Juliane: Luftkugel und fliegendes Auge. In: Stadler, Ulrich / Wagner, Karl (Hrsg.): Schaulust. Heimliche und verpönte Blicke in Literatur und Kunst. Paderborn 2005, S. 55-72.

Vogl, Joseph: Poetologien des Wissens um 1800. München 1999.

Wiebel, Bernhard: Münchhausen - das Märchen vom Lügenbaron. In: Bendix, Regina / Marzolph, Ulrich: Hören, Lesen, Sehen, Spüren: Märchenrezeption im europäischen Vergleich. Hohengehern 2008, S. 47-74.

Germanica Wratislaviensia 140, 2016

(C) for this edition by CNS 


\title{
Abstracts
}

Im Aufsatz wird untersucht, wie die Naturforschung (am Beispiel der aerostatischen Lehre) die literarische Produktion um 1800 beeinflusste. Hierzu erfolgt eine Auswertung von Bürgers Viertes Seeabenteuer, in welchem die Begegnung zwischen dem Baron Münchhausen mit einem französischen Wasserstoffballonfahrer beschrieben wird. Die Analyse legt einen Fokus auf die räumliche und zeitliche Ebene des Textes und zeigt - entgegen anderslautenden literaturwissenschaftlichen Meinungen - auf, dass in Bürgers Fassung durchaus Elemente der Naturforschung auf die erzählerische Ebene übertragen wurden. Insbesondere spiegelt sich die Struktur des naturwissenschaftlichen Experiments im literarischen Text auf mehreren Ebenen wieder.

Schlüsselwörter: Wissenschaftsgeschichte, um 1800, Transfer zwischen Literatur und Naturwissenschaft, Aerostatik, Ballonfahrten, Textanalyse, Münchhausen, Gedankenexperiment

\section{The influence of aerostatics on literature or the scientific potential of the "Münchhausiade"}

The aim of this essay is to show how natural sciences (in particular aerostatics) influenced literary production at the turn of the 18th century. The fourth sea-adventure of Münchhausen by Bürger serves to illustrate this intersection between science and literature. By analyzing the narrative structure (for instance, with regard to time and place of the setting) of this literary piece, I will demonstrate that the structure of the scientific experiment - especially the enlargement and reduction of objects - appears in Münchausen's fourth sea-adventure on several levels. As I will argue, the narrative can therefore be understood as a thought-experiment.

Keywords: history of science, 18th century, intersection between science and literature, aerostatics, Baron Munchhausen, narrative structure, thought experiment

\author{
Aleksandra Wojnarowska \\ Ernst-Moritz-Arndt-Universität \\ Institut für Deutsche Philologie \\ Rubenowstraße 3 \\ 17489 Greifswald \\ Deutschland \\ E-Mail: aw114427@uni-greifswald.de
}

